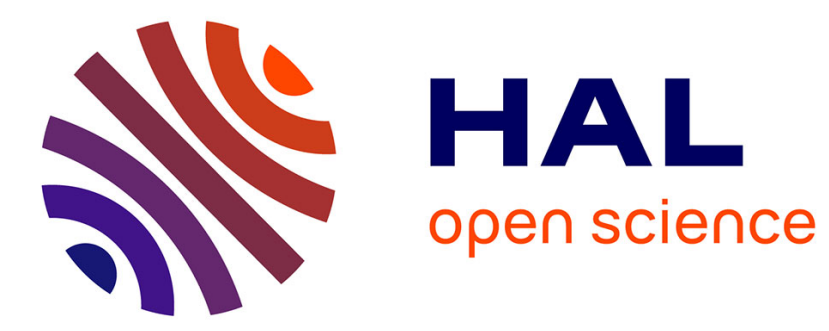

\title{
Drone Package Delivery: A Heuristic approach for UAVs path planning and tracking
}

Mustapha Bekhti, Nadjib Achir, Khaled Boussetta, Marwen Abdennebi

\section{To cite this version:}

Mustapha Bekhti, Nadjib Achir, Khaled Boussetta, Marwen Abdennebi. Drone Package Delivery: A Heuristic approach for UAVs path planning and tracking. EAI endorsed transactions on Internet of Things, 2017, 3 (9), pp.e1. 10.4108/eai.31-8-2017.153048 . hal-02084671

\section{HAL Id: hal-02084671 \\ https://hal.science/hal-02084671}

Submitted on 29 Mar 2019

HAL is a multi-disciplinary open access archive for the deposit and dissemination of scientific research documents, whether they are published or not. The documents may come from teaching and research institutions in France or abroad, or from public or private research centers.
L'archive ouverte pluridisciplinaire HAL, est destinée au dépôt et à la diffusion de documents scientifiques de niveau recherche, publiés ou non, émanant des établissements d'enseignement et de recherche français ou étrangers, des laboratoires publics ou privés. 


\title{
EAI Endorsed Transactions \\ on Internet of Things
}

\section{Drone Package Delivery: A Heuristic approach for UAVs path planning and tracking}

\author{
Mustapha Bekhti ${ }^{1, *}$, Nadjib Achir ${ }^{1}$, Khaled Boussetta ${ }^{2}$ and Marwen Abdennebi ${ }^{1}$ \\ ${ }^{1}$ Univ ersité Paris 13, Sorbonne Paris Cité - L2TI (EA 4303), France \\ ${ }^{2}$ INRIA URBANET, INSA Lyon, F-69621, Villeurbanne, France
}

\section{Abstract}

In this paper we propose a new approach based on a heuristic search for UAVs path planning with terrestrial wireless network tracking. In a previous work we proposed and exact solution based on an integ er linear formulation of the problem. Unfortunately, the exact resolution is limited by the computation complexity. In this case, we propose in this paper a new approach based on a heuristic search. More precisely, a heuristic adaptive scheme based on Dijkstra algorithm is proposed to yield a simple but effective and fast solution. In addition, the proposed solution can cover a large area and generate a set of optimum and near optimum paths according to the drone battery capacities. Finally, the simulation results show that the drone tracking is sustainable even in noisy wireless network environmen t.

Received on 08 March 2017; accepted on 25 August 2017; published on 31 August 2017

Keywords: UAV, Tracking, WSN, SINR, RPR, Noise, heuristic

Copyright (c) 2017 Bekhti et al., licensed to EAI. This is an open access article distributed under the terms of the Crea tive Commons Attribution license (http://creativecommons.org/licenses/by/3.0/), which permits unlimited use, distribution and reprod uction in any medium so long as the original work is proper ly cited.

doi:10.4108/eai.31-8-2017.153048

\section{Introduction}

For decades, Unmanned Aerial Vehicles (UAVs) are widel $y$ used in modern warfare for surveillance, reconnaissance, sensing, battle damag e assessmen $t$ and attacking. The benefit of UAVs include reduced costs and no warfighte risk. In fact UAVs use is increased by time, especiall $y$ under the concept of the netw orkcentric operation environmen $t$ and under the concept of revolution in military affairs. Recen tly, technol ogical advances in micro controllers, sensors, and batteries have drama ticall y increased their utility and versa tility and yet, a new horizon is open for civilian uses. This began with limited aerial patrols of the nation's borders, observation and aerial mapping, disaster response including search and support to rescuers, sports event coverage and law enforcemen t. Although the market is almost nonexisten $t$ today, this is most likel $\mathrm{y}$ in the civil fie $\mathrm{d}$ that drones are expected to play the larg est role. Recen tly, those f ying machines

\footnotetext{
$\star$ Please ensure that you use the most up to date class file available from EAI at http://doc.eai.eu/publications/transactions/ latex/

${ }^{*}$ Corresponding author. Email: bekhti.m ustapha, nadjib.achir, khaled.boussetta, marwen.abdennebi@univ -paris13.fr
}

have also been destined to the commercial market and have gained much attention. In fact, a forthcoming plans for commercial drone use have been recently announced by a number of companies around the world such, Amazon, Wallmart, DHL, and Zookal which are investing in mini drones developmen $t$ for variety of tasks, including freight and packag e deliv ery to consumers. The introduction of drones in civil applica tions raises new challeng es to the governmen $t$ authorities in charge of fligh security and air traffic manag emen $t$ which have to balance safety and public concerns against the poten tial economic benefit

By virtue of their small size, mini drones are difficult to be detected and to be tracked. In this frame, the European Parliamen $t$ adopted a resolution on the use of drones, which requires Member States to implemen $t$ various regula tions to ensure the saf ety of the airspace and to ensure the privacy of citizens threa tened by the use of these $f$ ying machines. Through this resol ution, it is considered that regardless of their sizes, the question of iden tifying is essen tial, and emphasized the need to provide appropria te solutions in terms of locating and tracking. In other words, this new report aims to ensure the traceability of all UAVs, but also oper ators and owners as a sine qua non-conditions for any use. 


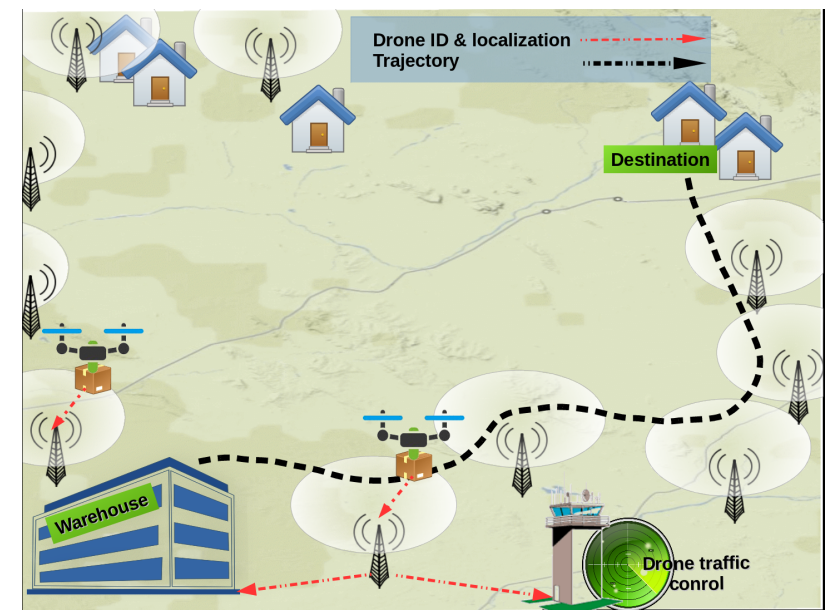

Figure 1. Drone package delivery

It is obvious that path planning is one of the most crucial tasks for mission definitio and manag emen $t$ of the aircr aft and it will also be an importan $t$ requiremen $t$ for UAVs that has autonomous fligh capabilities [1]. Basicall $y$, an efficien t off-line path planning could help to ensure a permanen $t$ localiza tion and tracking of the drone. Moreover, the predetermined trajectory unable to avoid obstacles and eventual collisions with other drones, and also to optimize certain functionalities in certain environmen t. However, mission nature, battery capacity, drone characteristics and hovering capabilities strongly inf uence the path planning strategy [2]. The oper ational problem that this work address is enabling the governmen $t$ authorities in charg e of fligh safety to iden tify, locate and to track drones. Usuall y the area is larg e and the detection and localiza tion time to fin the UAV is the critical parameter that shoul d be minimized.

To this end and in order to make this possible, we presen $t$ in this paper a newly approach based on the expl oitation of the available wireless netw ork coverage. This approach relies on a powerful interaction, or collabor ation between the UAVs and the operators. Cooperation in such environmen $t$ implies that the drone periodicall y send its iden tific tion, localiza tion, speed and other information to the remote operators through the available wireless netw orks. The solution we aim to presen t provide or inform of the optim um and the near optim um paths that the drone should follow to ensure a reliable communication and high packet deliv ery rate depending on its battery autonom y.

In our previous work [3], we formula ted the problem as an Integ er Linear Problem. Moreov er, we expressed in an analytic manner the packet loss rate of tracking messag es depending on the UAV location and the wireless netw ork coverage. By solving the ILP problem using CPLEX, we were being able to anal yze how the radio coverage as well as the threshol $\mathrm{d}$ on the packet success rate, impact the number of possible solutions and the trajectory of the UAV. Unfortuna tely, due to the computational complexity the proposed approach was not able to provide a path planning solution for a larg e area. In addition, the packet success rate was computed by considering only the radio channel and without any MAC layer oper ations.

Our curren t investig ations focus on the complexity issue raised for larger area size. For the drone path planning, a heuristic adaptiv e scheme based on Dijkstr a algorithm is presen ted to cope with the problem of scalability. The fligh path of drone is optimized in order to improve its connectivity to the available terrestrial wireless netw ork and consequen tly its localiza tion, iden tific tion and tracking. Moreover, the solution is proposed to yiel d a simple but effective and fast solution and tested under a more realistic scenario char acterized with a noisy environmen $t$.

\section{State of the art}

Path planning for kinema tic system issues has been widel y studied and has been addressed using differen $t$ approaches and techniques. Thus, several approaches exist for computing paths given some input variables of the environmen $t$. In general, the two most popular techniques are deterministic, heuristic-based algorithms [4], [5], [6] and probabilistic, randomized algorithms [7] and [8]. The choice of the algorithm to use depends on the type of problem to be solved. Although, the robotic bibliogr aphy on this subject is very rich, it's not the case for the UAV's one.

For the autonomous fligh of drones, path planning is one of the most crucial and importan $t$ issues to solve. Now ada ys, the applica tion of UAV is extending from high-altitude fligh to very low-altitude, where the impact of the terrain, the environmen $t$ and the air traffic will be the key factors to be considered to avoid collisions [9]. How ever, we do not aim to provide an exha ustive list but we will be limited to provide the most relev ant work rela ted to the path planning regarding to the nature of the objectiv es, problems formaliza tion and resol ving methods.

The author in [10] presen ted a framew ork to compute the minim um cost cooper ative route betw een a heterog eneous packag e delivery team composed of a truck and micro drones. They abstracted the problem on a graph and formulate the issue as a discrete optimal path planning problem. In the same context of heterog eneous teams, the authors in [11] presen ted a path planning problem involving an UAV and a ground vehicle for intellig ence, surv eillance and reconnaissance missions. The addressed problem is similar to the ring-star problem and the hier archical ring netw ork problem.

On the other hand, the authors in [9] and [12] presen ted three dimensional path planning solutions 
for unmanned aerial vehicles. The firs solution is based on interfered $\mathrm{f}$ uid dynamic system, while the second approach uses linear progr amming where obstacle avoidance and targ et tracking are linearized to gener ate a linear progr amming model in a rela tive velocity space. Dealing with adversarial environmen ts, the authors in [13] and [14] presen ted solutions for unmanned aerial vehicles path planning in uncertain an adversarial environmen $t$ in sight to reach a given targ et, while maximizing the safety of the drone. They proposed a path planning algorithm based on threa ts probability map which can be built from a priori surveillance data and from a mechanism based on a predictiv e model control.

Another importan t work is [15], which contains concise summaries. It focused on dynamic problems and discussed a family of heuristic algorithms for path planning in real-world scenarios such as $A^{*}, D^{\star}, A R A^{*}$ and $\mathrm{AD}^{\star}$. Finally, it is worth mentioning the research done by [16] that can be considered one of the few papers dealing with path planning strategies destined for a based UAVs network. The authors compared deterministic and probabilistic path planning strategies for autonomous drones to expl ore a given area with obstacles and to provide an overview image. The resul ts show ed that, although the deterministic approach could provide a solution, it requires more knowledg e and time to generate a plan. However, the probabilistic approaches are flexibl and adaptiv e.

To the best of our knowledg e, none of the above works have investig ated UAV path planning problem assuming that the UAV uses terrestrial wireless netw orks to transmit its positions.

\section{Path planning problem formulation}

\subsection{Problem statement and system description}

In this paper, we are considering a packag e deliv ery service using UAVs. Basicall $y$, a UAV has to deliv er a packag e from a depot or warehouse to a predetermined destina tion or consumer. The main objectiv e of this paper is to provide an off-line path planning that aims to minimize the deliv ery dela y with respect to the UAV's resid ual energy constraint while ensuring an optim um tracking of the UAV's at the oper ator side.

In this frame, the system is modeled as $2 \mathrm{D}$ area $A$ without any obstacle. The projection of the $\mathrm{f}$ ying area is represen ted by a rectangular with length of $x_{\max }$ and a wid th of $y_{\max }$. We suppose that the drone $D_{\text {rone }}$ keeps the same altitude $h$ from the starting point $O$ to the destina tion $D$. A set of wireless receiv ers or Base Stations $B S=\left\{B S_{1}, B S_{2}, \ldots . B S_{n}\right\}$ is depl oyed randoml $y$ at differen $t$ altitudes in order to provide a wireless access infr astructure. In addition, we assume a partiall y noisy environmen $t$ with the existence of a certain number of noise nodes $N_{\text {oise }}=\left\{N_{N 1}, N_{N 2}, \ldots . . N_{N n}\right\}$ depl oyed within
$A$ and uses the wireless infrastructure as an access netw ork. We also consider that the drone has a limited fligh autonomy $\Upsilon$ and is equipped with a wireless interface in order to communica te with the other Base Stations. The latter has a short sensing range compared to the size of the region of interest. Moreover, we consider that $A$ is discretized into $C$ hexag onal cells of the same dimension. This implies discrete position for the UAV, which then is supposed to be located in the center of the considered cell. The transition cost betw een two neighbor cells depicts certain reliability of communica tion, i.e. a certain probability that the communica tion is not interrupted and has a specifie Reception Packet Rate RPR. In this paper, the OMNeT++ 4.61 simula tor and the INET framew ork were used to gener ate both the signalto-interf erence-pl us-noise ratio $S I N R$ maps and the Receiv ed Packet Rate for all possible transitions in $A$.

Our goal is to determine a path or a set of paths that maximize the drone localiza tion and tracking using a wireless netw ork, such as cell ular or IEEE $802.11 \mathrm{x}$ technol ogies. For this purpose, we assume that after each period $T$, the drone gener ates a messag e of size $d$ bits containing its iden tific tion, position and speed. The on-board wireless interface tries to send each gener ated messag e to the remote UAV monitoring and controlling system via the set $B S$ while the jamming nodes attem pt to overload the netw ork by sending messag es in a continuous and unpredictable manner to the BS. For that reason, a messag e can be corrupted or even lost due to possible interf erence and collisions. The opportunity to transmit also depends on the radio coverage, the capacity of the rela ted wireless technol ogy and the drone's location.

\subsection{Problem formulation}

In order to describe the proposed mathema tical model that represen ts the optim um path planning problem, it is useful to introduce the following notations and definitions

First, we model the problem with the help of a directed and valued graph $G$ consisting of $n$ hexag onal cells, where the valuation of an arc is comprised betw een 0 and 1 , indica ting the reception packet rate $(R P R)$ on that arc.

Finall $y$, we defin $c_{i j}$ the cost of using the arc going from cell $c_{i}$ to cell $c_{j}$. The fow going that way is denoted by a binary variable, noted as $x_{i j}$, where

$$
x_{i j}= \begin{cases}1, & \text { if the drone moves from cell } i \text { to cell } j \\ 0, & \text { otherwise. }\end{cases}
$$

The cost of a path represen ts its reliability and it is set to the product of the $R P R$ of each cell forming the resul ted path: 


$$
P a t h_{\text {cost }}=\prod_{i=1}^{n} \prod_{j=1}^{n} R P R_{i j} * x_{i j}
$$

As, the $R P R_{i j}$ is comprised betw een $\left.] 0,1\right]$, this means more we add a new cell to the path more the path cost is low. Thus, the firs two objectiv es for our drone path planning problem are reported as follows:

$$
\operatorname{minimize} \sum_{i \in A} \sum_{j \in A} c_{i j} x_{i j}
$$

and

$$
\operatorname{maximize} \prod_{i=1}^{n} \prod_{j=1}^{n}\left(R P R_{i, j}\right) x_{i j}
$$

where, as define earlier, $c_{i j}$ is the cost of the arc going from cell $c_{i}$ to cell $c_{j}$. In this paper, we consider $c_{i j}$ as the amoun $t$ of energy consumed by the drone on that arc,

The objective functions (3) and (4) represen $\mathrm{t}$ respectiv ely the minimiza tion of the energy consumed by the drone and the maximiza tion of the tracking probability between the start point $O$ and the destina tion D. Basicall $y$, we should fin the shortest possible path, in terms of consumed energy, that passes through the cells with highest Receiv ed Packet Rate, see Fig 2.

In addition to the last two objectiv es, we also add a third objectiv e that aims to minimize the tracking time loss of the drone, by avoiding passing through several adjacen t cells with low $R P R$. For exam ple, as ill ustr ated in Fig 3, if we have to choose between the path $a(0.9$, $0.9,0.9,0.1,0.1,0.1)$ and the path $b(0.9,0.1,0.9,0.1$, $0.9,0.1)$ with the same length and the same average packet deliv ery ratio, than we have to privileg e the solution $b$ rather than $a$. The privileg e of the solution $b$ is motivated by the fact that we have fewer adjacen $t$ cells with low packet deliv ery probability. The main benefi of this choice is to have the communication rupture spaced out on the time rather than having a long time with no comm unica tions.

To this end, we need to anal yze the cells data in terms of $R P R$ values and their positions in the path by creating series of averages of different subsets of the full path. Basicall y, given $K$ a path and the subset size equals to 2 , the firs elemen $t$ is obtained by taking the average of the two initial adjacen $t$ cells of the selected path. Thereafter, the subset is modifie by shifting it forward, excluding the firs cell and including the next cell in $K$. This creates a new subset of numbers $\bar{K}$. This kind of mathema tical transformation is also used in the signal processing in order to mitig ate the higher frequencies and to retain only the low frequencies or the contrary.

The principle of averages on a shifted window is interesting in the case when we use prediction

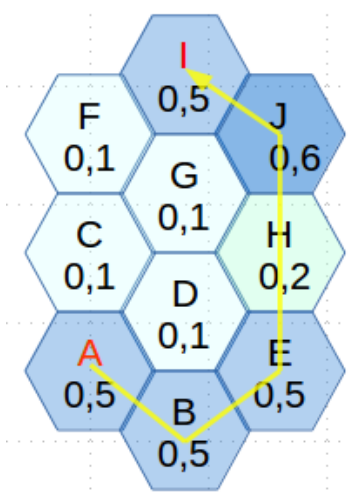

Figure 2. Example of a path from the origin $A$ to the destination I where the shortest path with high packet delivery rate is $(A, B$, $E, H, J, I)$

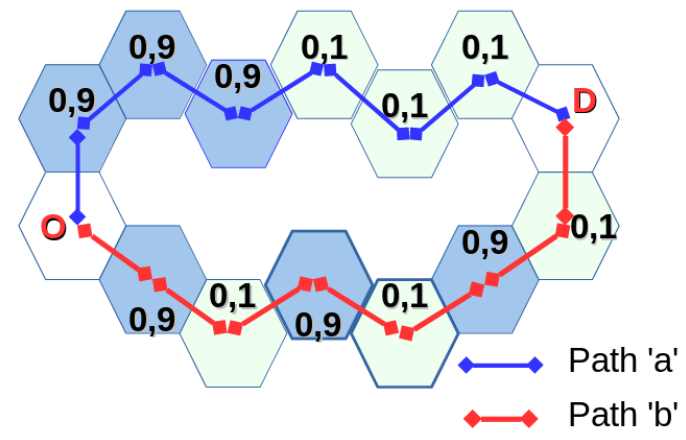

Figure 3. example of paths with the same cost

algorithms. Basicall y, we need to compute an average data based on the most recen $t$ results in order to crea te forecasts. Indeed, the most recen $t$ data are more importan $t$ or more meaningful than older data. Let's consider $f(K)$ the score function and $K$ is the path to anal yze, where $K=\left\{R P R_{1} ; R P R_{2} ; \ldots . R P R_{n}\right\}$ with $R P R_{1}$, $R P R_{2}, \ldots R P R_{n}$ are the Receiv ed Packet Rate at the cells $c_{1}, c_{2}, \ldots c_{n}$ forming the path $K$ and $\bar{K}=\left\{\bar{K}_{1} ; \bar{K}_{2} ; \ldots . \bar{K}_{n-1}\right\}$, where $\bar{K}_{i}=\left(R P R_{i}+R P R_{i+1}\right) / 2$.

Since the geometric average is less sensitiv e than the arithmetic average to the highest or lowest values of a series, we propose the following cost function:

$$
f(K)=\sqrt[n-1]{\prod_{i=1}^{n-1} \bar{K}_{i}}
$$

Thus, by applying the formulas 5 on the previous paths $\quad a=\{0.9,0.9,0.9,0.1,0.1,0.1\}$ and $b=\{0.9,0.1,0.9,0.1,0.9,0.1\} \quad$ we will get: $\bar{a}=\{0.9,0.9,0.5,0.1,0.1\} \quad$ and $\quad f(a)=0.33$, and $\bar{b}=\{0.5,0.5,0.5,0.5,0.5\}$ and $f(b)=0.5$. Since we need to maximize the function $f$, the path $b$ will be selected. 
Finall $y$, in addition to the last objectiv es, we add a new constr aint rela ted to the UAV's maximal fligh distance:

$$
\sum_{i \in A} \sum_{j \in A} c_{i, j} x_{i j}<\delta,
$$

where $\delta$ is the maxim um energy that the UAV could have.

\subsection{Path computation}

Differen $t$ shortest path algorithms exist like $A^{*}$, Dijkstr a, Bellman-F ord and others. Our proposal is based and adapted from Dijkstr a algorithms. The latest is one of the most common and effective algorithms used to search the shortest path betw een two vertices are in a graph in terms of distance. For our case, we adapt the Dijkstr a algorithm to fin the shortest path with high communica tion reliability and high packet reception.

As introduced in our problem formulation section, our objectiv es are firs to minimize the traveling distance and to maximize the tracking probability betw een the start poin the destina tion poin $t$. The firs objectiv e correspond to the classical Dijkstr a algorithm. On the other hand, for the second objective we are dealing with probabilities. We have to fin the shortest path where the prod uct of the probabilities $R P R_{i}$ of the visited cells that constitute a given path is maximized. More over, each time a cell is added to a path, the product of the probabilities decreases. In this case, our algorithm firs starts by initializing the cost of the origin cell $c_{o}$ to 1 . The cost of the remaining cells is set to 0 . Starting from the origin point, we buil t step by step a set of $P$ marked cells. For each marked cell $c_{i}$, the cost is equal to the prod uct of the Receiv ed Packet Rate probabilities of all predecessors cells. At each step, we select an unmar ked vertex $c_{j}$ whose cost is the highest among all vertexes not marked, then we mark $c_{j}$ and we upda te from $c_{j}$ the estima ted costs of unmarked successors of $c_{j}$. We repea $t$ until exha ustion unmar ked vertexes.

In addition to the above algorithm, we also derived a set of near optimal paths. In fact, the solution was extended to compromise localiza tion data deliv ery rates and distance between the starting point and the destina tion with the respect of the drone autonom y. To this end, if the length of the optimal path is grea ter than the drone autonomy or simply, the operator would to have multiple choice of short paths, then we re-execute the function above until we get the desired solution and for each execution we set the RPR of the cells of the obtained path to $\epsilon$, where $\epsilon$ is a small non-null value. This allows us to generate a new path totall $y$ differen $t$ from the previous one. All these paths can then be compared using the cost above function $f$ for a better drone tracking resul t.
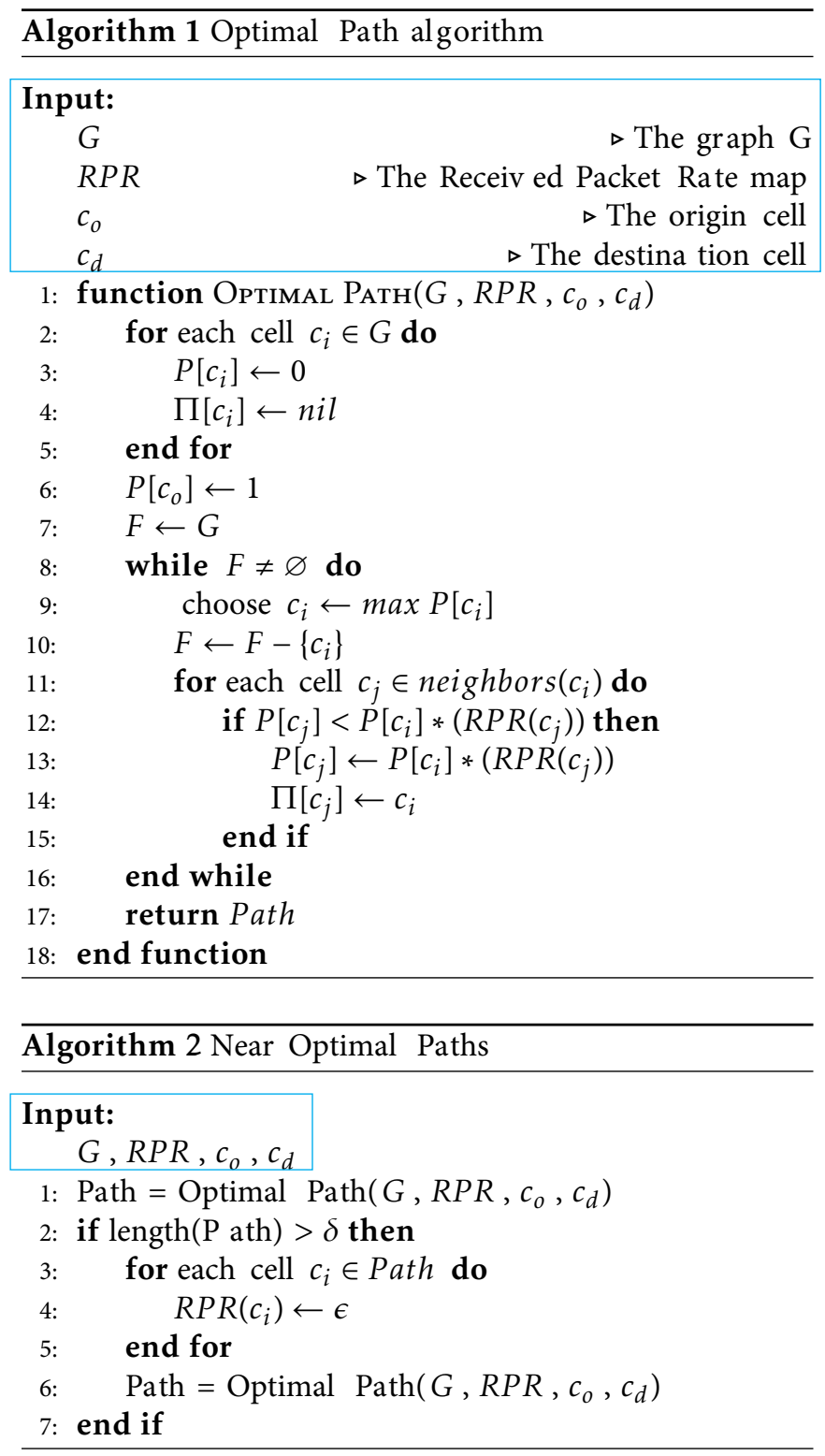

\subsection{Energy Consumption Model}

In this section we estima te the energy consumed by each drone according to its char acteristics.

The main challeng e for the construction of rotary wing drones is to maximize its autonom y for a given mass, while providing the pow er needed for propulsion and for the embedded instrumen ts. It is theref ore importan t to carefull y manag e the available energy and the path planning with each other to have the best overall. In fact, recen $t$ progress achiev ed on Lithium battery type allowed the electric fligh to achiev e a reall $y$ interesting autonom $y$ for entertainmen $t$ or local missions, but still far from being effective for longer trips and missions. 
In this paper, we consider a quad-copter which is a drone with four rotors at the ends of a cross. The four rotors provide the vertical force (Thrust) that allows the unit to rise. In fligh, the quad-copter may evolve following its roll, pitch and yaw axes and also in transla tion in all directions, fi 4 . Basicall $y$, the dynamic model of quad-rotor can be seen as a system where the spatial evolution's are the outputs and the voltage of each engine are the inputs, fi 5. Motion is achiev ed by changing the rotation speed of one rotor or more. Thus, to control the roll of the UAV, it is sufficient to act on the rotational speeds of the motors 2 and 4 . In the same way, the pitch of the UAV is controllable by acting on the motors 1 and 3 .

Furthermore, main taining a stable fligh requires an equilibrium and a balance of all forces acting upon a drone. Weight, lift, thrust and drag are the acting forces on a drone. These Forces are vector quan tities having both a magnitude and a direction and consequen tly, the motion of the drone through the air depends on the rela tive magnitude and direction of these forces. A gener al derivation of the thrust force equa tion shows that the amoun $t$ of thrust generated depends on the mass $f$ ow through the rotors and the chang $e$ in rotation speeds of the four propellers.

In fact, several methods exist in the liter ature all owing to have an order of magnitude of the pow er of a propeller, such as the blade elemen $t$ theory (BET) and the Froude theory. Even if these methods can provide a more precise result, they are based on a certain number of coefficien ts which cannot be computed only after empirical tests, like Thrust Coefficient, Torque Coefficien $t$, Power Coefficien $t$, etc... In addition, the obtained coefficien ts are specifi to the tested propeller at a specifie rotation speed and cannot be used for other types of propellers. Basic drone manoeuvres include take-o ff, hovering, changing altitudes, and landing. This manoeuvre requires different rotors and propeller rotation speeds. To our knowledg e, the best method to approxima te drone pow er consum ption is to use formulas that connect pow er to rotor rotation speed, propeller diameter and pitch like the one proposed by Abbott, Young, Boucher, and Aguerre.

As ill ustrated in figur $4, \Omega_{1}, \Omega_{2}, \Omega_{3}, \Omega_{4}$ are the rotation speed of the propellers; $T_{1}, T_{2}, T_{3}, T_{4}$ are the Forces gener ated by the propellers; and final $\mathrm{y} m g$ is the weight of the quadrotor;

In the following, the Boucher formula is used. In fact, the latest was used to compute the fligh autonom y and the power consum ption for a real quadcopter drone type of Phantom 3 Advanced. The resul ts were very close to the ones presen ted by the man ufacturer:

$$
P_{p}=K *\left(\frac{\text { Diam }}{12}\right)^{4} * \frac{\text { Pitch }}{12} *\left(\frac{N_{t}}{1000}\right)^{3}
$$

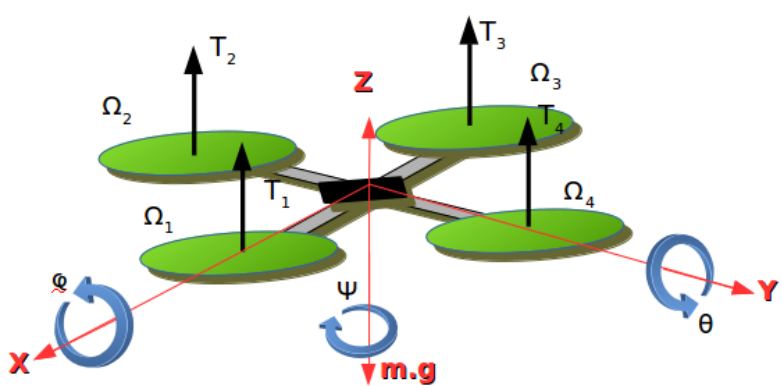

Figure 4. Dynamics involved in the quadcopter

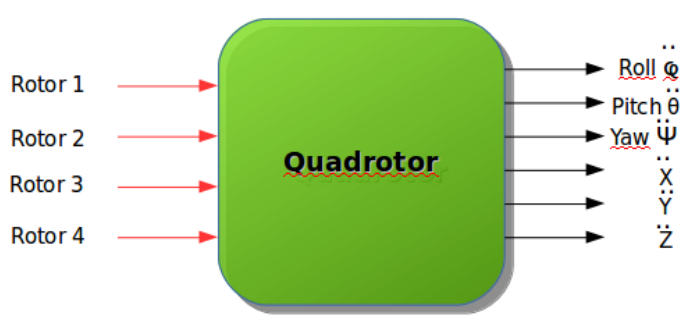

Figure 5. Dynamic Model of quadrotor

with $P_{p}$ in Watt, Diam and Pitch in inch, and $N_{t}$ in $t r / m n . K$ is an adjustmen $t$ parameter, which depends on the propeller type, (APC: 1.11, Graupner: 1.18, Zing er: 1.31, Top flite 1.31, etc..).

To link the aerodynamic properties of the propeller to the pow er and the engine speed, we will need three formulas:

- the pow er supplied by the propeller $P_{p}$ in watts;

- the thrust of the propeller in $\mathrm{Kg}$ :

$$
T_{p}=4.9 * \text { Diam }^{3} * \text { Pitch } * N_{t}^{2}
$$

- and the speed of air passing through the propeller of in $\mathrm{Km} / \mathrm{h}$ :

$$
S_{\text {air }}=60 * \text { Pitch } * N_{t}
$$

where Diam is the propeller diameter in meter, Pitch in meter and $N_{t}$ is the number of thousands revolutions per minute (rpm). In addition to the last formulas, we also need to compute:

- The pitch:

$$
\text { Pitch }=\pi * \operatorname{Diam} * \operatorname{Tang}(\alpha),
$$

- The power consumed by the propeller

$$
P_{C}=P_{p} * C_{e} * R_{e}
$$




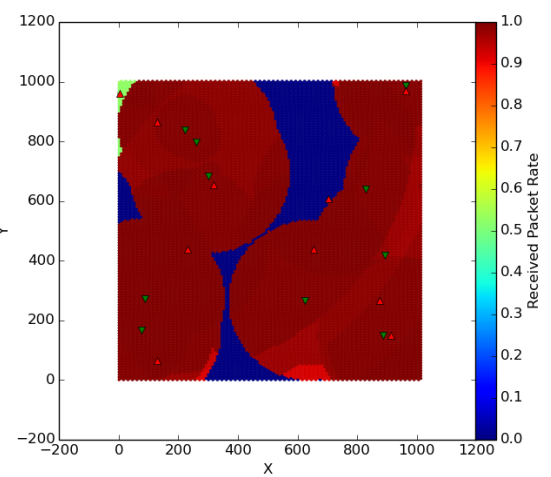

Figure 6. RPR with 10 noise nodes at $h=60 \mathrm{~m}$

- The drone fligh end urance can be expressed as in [17], and by ignoring the consumed power at the idle sta te we get:

$$
F_{\text {Endurance }}=B_{C} / P_{C}
$$

where $R_{e}$ and $C_{e}$ are the rotor efficiency and the controller efficiency, gener ally fixe at $75 \%$ and 98\% respectiv ely, $\alpha$ is the attack angle of the propeller, $B_{C}$ the Capacity of the battery, $P_{C}$ is the Power consumed by the propeller .

Since the power is the rate of doing work, it is equiv alen $t$ to the amoun $t$ of energy consumed per unit time. If work is done quickl y, more pow er is used and if work is done slowly, very little pow er is used. Thus, the energy consumed by the propellers to ensure the thrust forces required for the fligh can be expressed as:

$$
E_{M v m t}=\int P_{C}(t) d t
$$

Finall y, using the last equa tion we can derive the energy $c_{i j}$ required for a drone to $\mathrm{f}$ y from cell $i$ to cell $j$.

\section{Results}

In this section, we evaluate our proposed algorithm. Two main objectiv es were fixed firs, to ensure a maxim um tracking of the drone along with its fligh while the second one was to minimize the energy required to travel along the path in accordance with the drone fligh autonom y and the capacity of its battery. In addition to the last objectiv es we also consider a third objectiv e which is to minimize the number of adjacen $\mathrm{t}$ cells with low $R P R$.

In this case, we assess our proposed the algorithm in case of different scenarios. We start, using the OMNET++ simulator, by generating the $R P R$ map for a given altitude and in the presence of a given number of nodes using the wireless netw ork. Basicall $y$, in order to increase the packet losses we can increase the altitude of

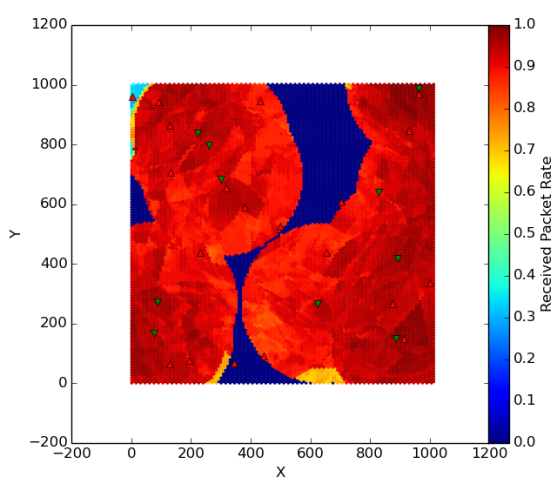

Figure 7. RPR with 20 noise nodes at $h=60 \mathrm{~m}$

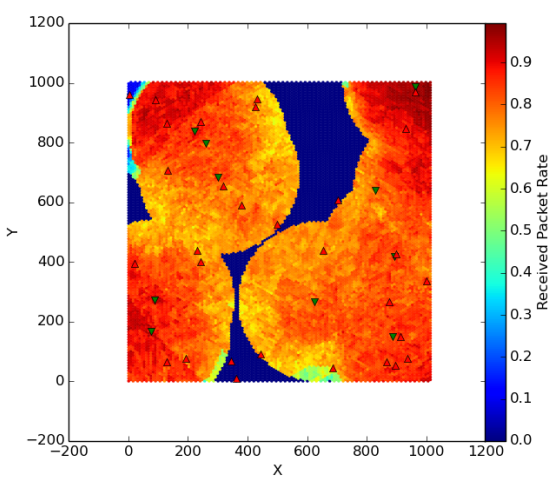

Figure 8. RPR with 30 noise nodes at $h=60 \mathrm{~m}$

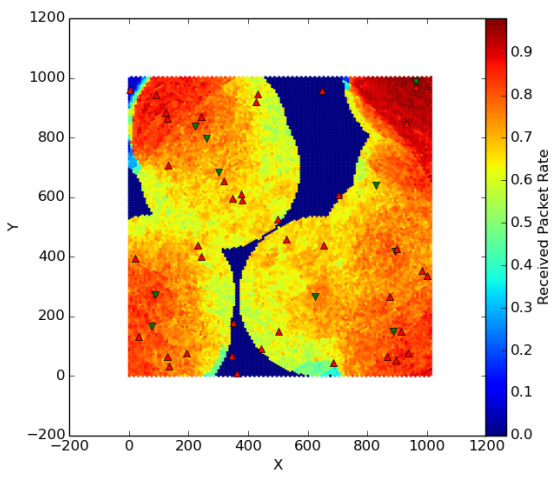

Figure 9. RPR with 40 noise nodes at $h=60 \mathrm{~m}$

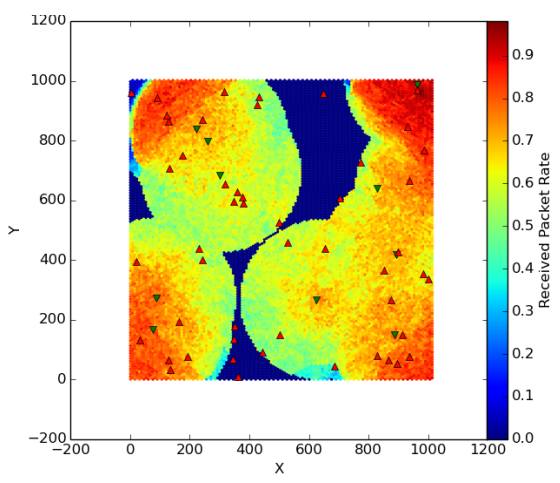

Figure 10. RPR with 50 noise nodes at $h=60 \mathrm{~m}$ 
Table 1. Simulation parameters

\begin{tabular}{|l|l|}
\hline Area & $\mathrm{X}=\mathrm{Y}=1000 \mathrm{~m}$ \\
\hline Cell radius (constan $\mathrm{t})$ & $\mathrm{a}=5 \mathrm{~m}$ \\
\hline BSs & 10 \\
\hline Noise nodes & $10,20,30,40,50$ \\
\hline UAV al titude & $60 \mathrm{~m}$ \\
\hline$D$ & 200 bytes \\
\hline$P_{t}$ & $20 \mathrm{dBm}(100 \mathrm{~mW})$ \\
\hline Background noise pow er & $-72 \mathrm{dBm}$ \\
\hline Path loss type & Two Ray Ground Ref. \\
\hline Antennas Gains & $\mathrm{Ge}=\mathrm{Gr}=10 \mathrm{dBi}$ \\
\hline Carrier Frequency & $2.4 \mathrm{GHz}$ \\
\hline
\end{tabular}

the drone or the number of nodes acting as noisy nodes. In the following, we provide some resul ts according to the simulation parameters summarized in the table 1 .

For an ideal environmen $t$ with no interference and noise, the drone shall fligh closer from the BS station to ensure a permanen $t$ tracking and localiza tion. How ever, this is not the case in reality. Thus, the figure $6,7,8,9$ and 10 illustrates the receiv ed packet rate in a noisy environmen $t$. It shows clearly that more noise nodes (red dots) are presen t more we have low $R P R$. We can also notice that for the receiv ed packet rate, the resul ts are better in the edge of the area, this even for the same $S I N R$. This can be explained by the fact that these subareas are less subject to physical radio errors beca use of the position of the receiv er who will experience fewer physical collisions and busy channel state.

Figures $11 \mathrm{a}$ and $11 \mathrm{~b}$ represen $\mathrm{t}$ respectiv ely the shortest path with highest $R P R$ at $60 \mathrm{~m}$ of al titude with the presence of 20 and 50 noise nodes. We compared our algorithm to the shortest path using the well-known Dijkstr a algorithm since to the best of our knowledg e there is no other work similar to our work in the liter ature. We also ill ustrate, in figure $11 \mathrm{c}$, the set of paths that we generated by our algorithm to compute the optimal path and optimizing our third objectiv e.

To understand the impact of increasing the interferences on the path length and $R P R$, we varied the number of the nodes simula ting the noisy environmen $t$. We set the drone altitude to $60 \mathrm{~m}$ and we measure the length of the optimal paths and their respectiv e RPRs. As we can observe in figur 12 and 13, if we increase the number of noise nodes, we gradually decrease the quality of the signal and subsequen tly the $R P R$ and the path length also decrease. In fact, in case of good radio coverage, the drone tends to be attracted to the cells with higher $S I N R$, which represen the $B S$ locations. On the other hand, when we degr ade the $S I N R$, the drone tends to take the shortest path to its destina tion.

In order to evaluate the efficiency of our solution, we tested the proposed algorithm for a thousand random destina tion points in an environmen $t$ with low signal

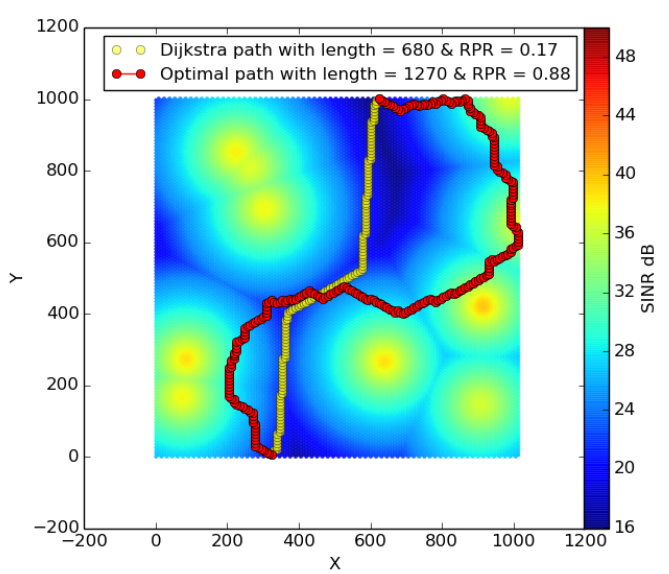

(a) noise nodes $=20$

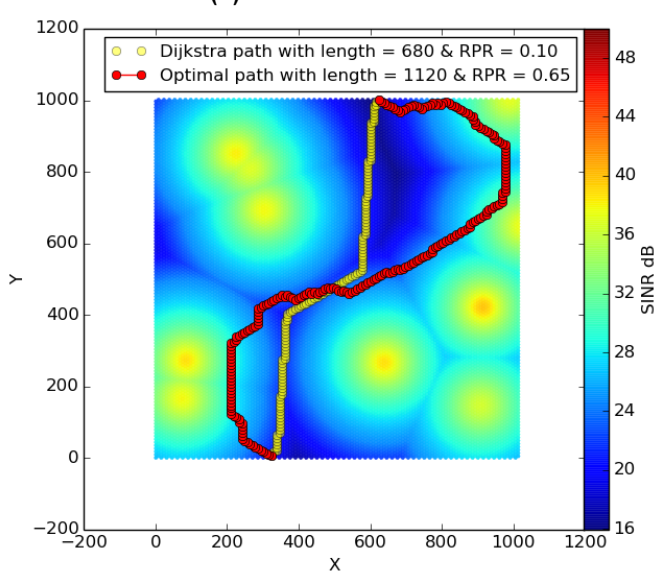

(b) noise nodes $=50$

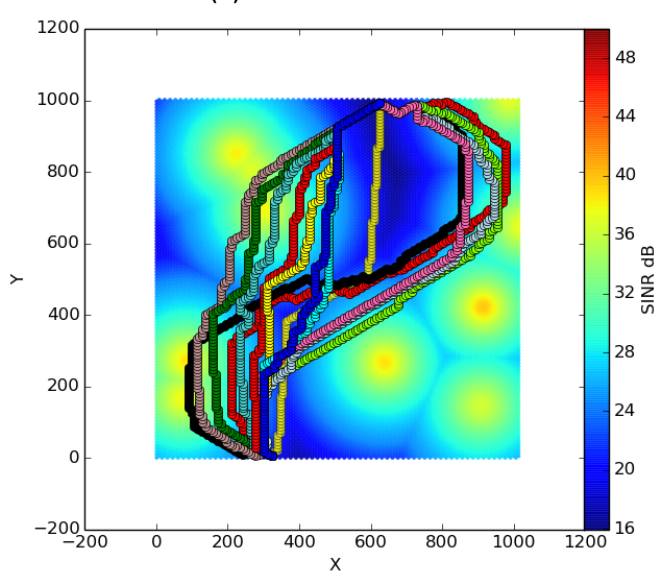

(c) set of near Optimal paths

Figure 11. Optimal and near optimal paths, $h=60 \mathrm{~m}$

coverage by setting the number of noise nodes to 50 nodes and the drone altitude to $60 \mathrm{~m}$. We compare for all these poin ts the resul ted paths with Dijkstr a's short path in terms of length and $R P R$. The comparisons are ill ustr ated in the figure 14 and 15 . Indeed, the gap is importan $\mathrm{t}$ in term of $R P R$. For the computed paths, the 


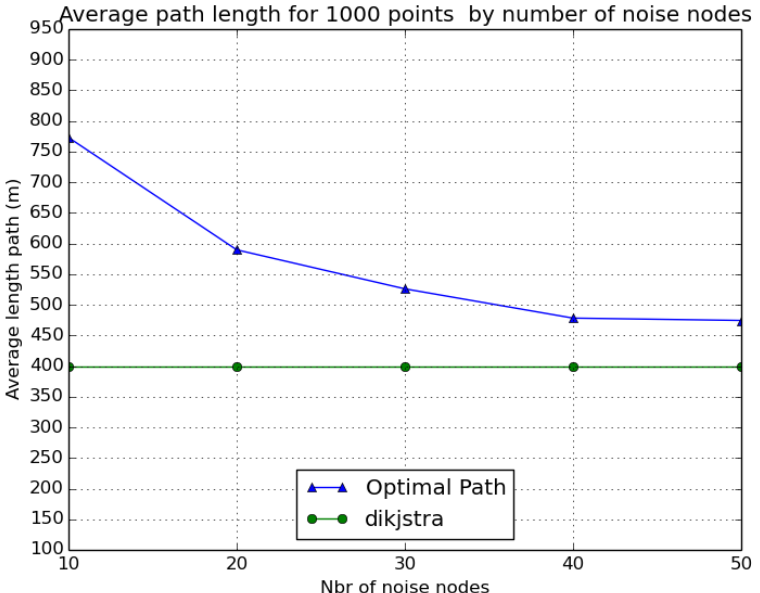

Figure 12. Path lengths with different number of noise nodes, $\mathrm{h}=60 \mathrm{~m}$

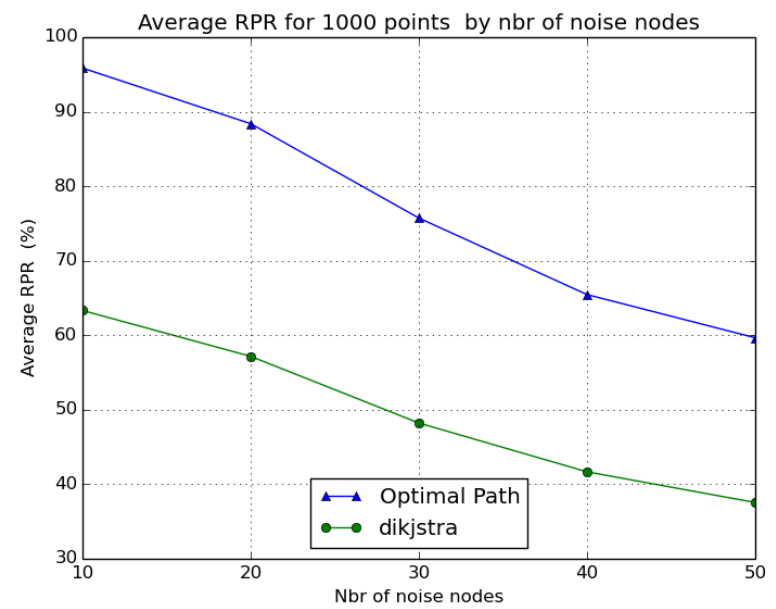

Figure 13. Received Packets Rate with different number of noise nodes, $h=60 \mathrm{~m}$

difference varies from 0.15 to 0.55 even for a path with length closer to Dijkstr a short path length.

In figure 16 and 17 we ill ustrate the impact of the drone speed on the packet receiv ed rate and the consumed energy. The resul ts were obtained using the Omnet++ simula tor. We vary the drone speed from $10 \mathrm{~m} / \mathrm{s}$ to $18 \mathrm{~m} / \mathrm{s}$, which are the most common drone speeds, and we compare the simula tor resul ts to the theoretical ones. We can notice that the $R P R$ remains almost the same for drone speed varying from $10 \mathrm{~m} / \mathrm{s}$ to $13 \mathrm{~m} / \mathrm{s}$. However, this rate decrease once the drones exceed the speed of $14 \mathrm{~m} / \mathrm{s}$. Almost $10 \%$ of the tracking capability is lost due to the drone's speed. In addition to the same payload, a drone will consume about a double in terms of energy when increasing the speed from $10 \mathrm{~m} / \mathrm{s}$ to $18 \mathrm{~m} / \mathrm{s}$. This consum ption is due to the increased rotational speed of the propellers.

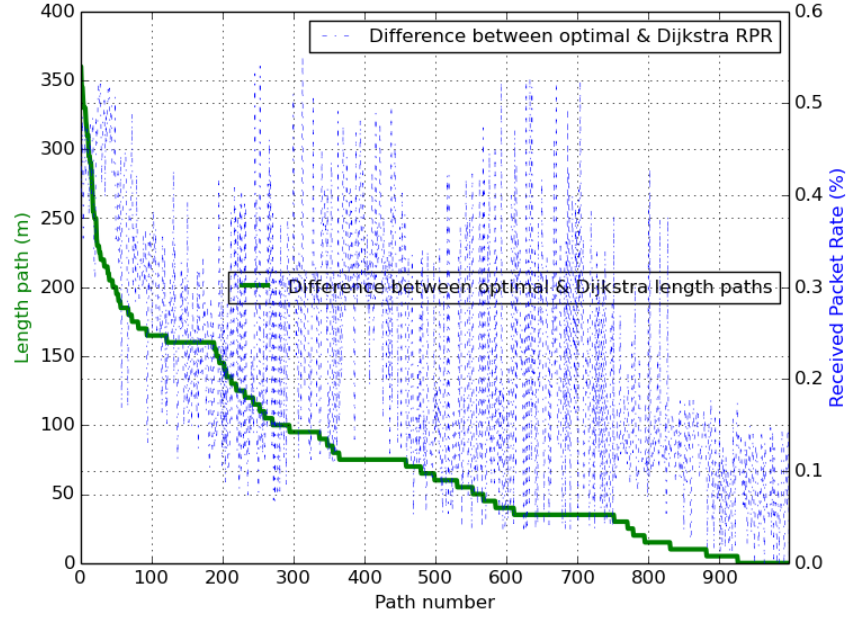

Figure 14. Difference between optimal and Dijkstra path length, nbr paths $=1000$

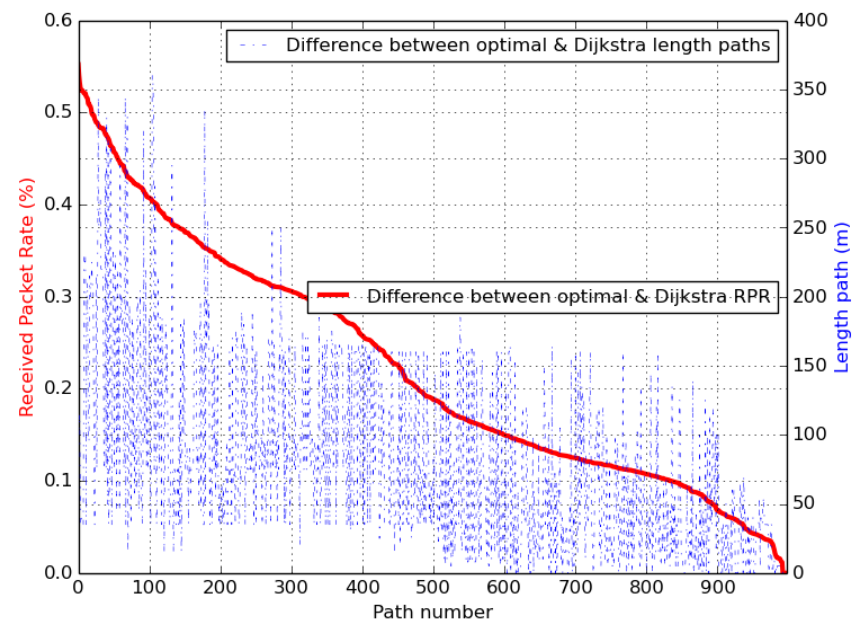

Figure 15. Difference between optimal and Dijkstra RPR, nbr paths $=1000$

Finall y, the figur 18 summarizes and ill ustrates clearly the advantage of our proposal in terms of drone localiza tion and tracking. In fact for tw o drones starting from the same poin $\mathrm{t}$ and $\mathrm{f}$ ying to the same destina tion at the same altitude, the capacity of tracking the drone at the controller side is different. As we can see, the tracking capability of the drone following the path generated by our algorithm reaches $88 \%$, while for the one following the Dijkstr a shortest path the tracking capability is about $14 \%$.

\section{Conclusion}

In this paper, we propose a path planning algorithm for UAV. Our approach doesn't only gener ate one single 


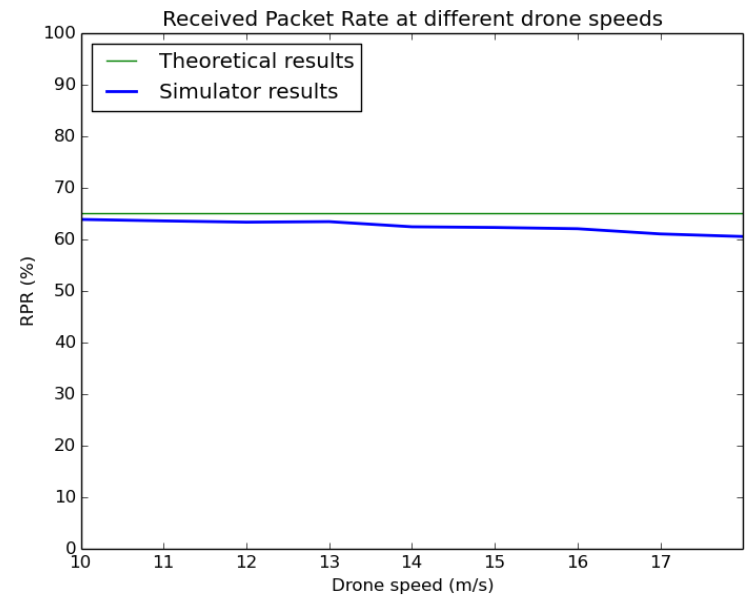

Figure 16. Received Packet Rate at different drone speeds, noise nodes $=50$

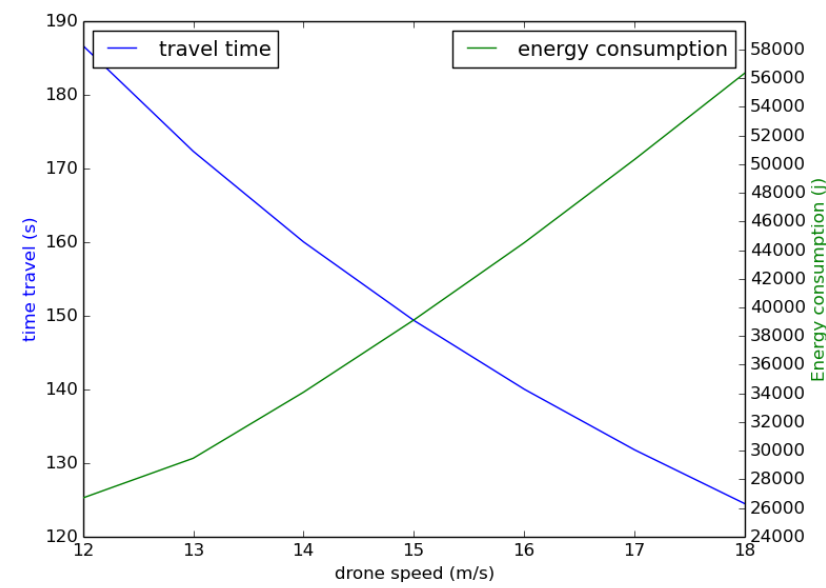

Figure 17. Energy consumption at different drone speeds

optimal solution but a number of other near optimal paths with a trade-off between length distance and probability of localiza tion determined by the drone fligh autonom y. Theref ore, we choose the best path suited to the need of localiza tion and tracking but also to the capability of the UAV in terms of energy autonom y. More precisel y, if iden tific tion, localiza tion and tracking are the main concerns than we can choose the longer path which insures a high communication probability and if the UAV energy autonomy is a priority than the we need to choose the suitable path length according to the battery duration.

Torqua y, Dev on, UK. Website:

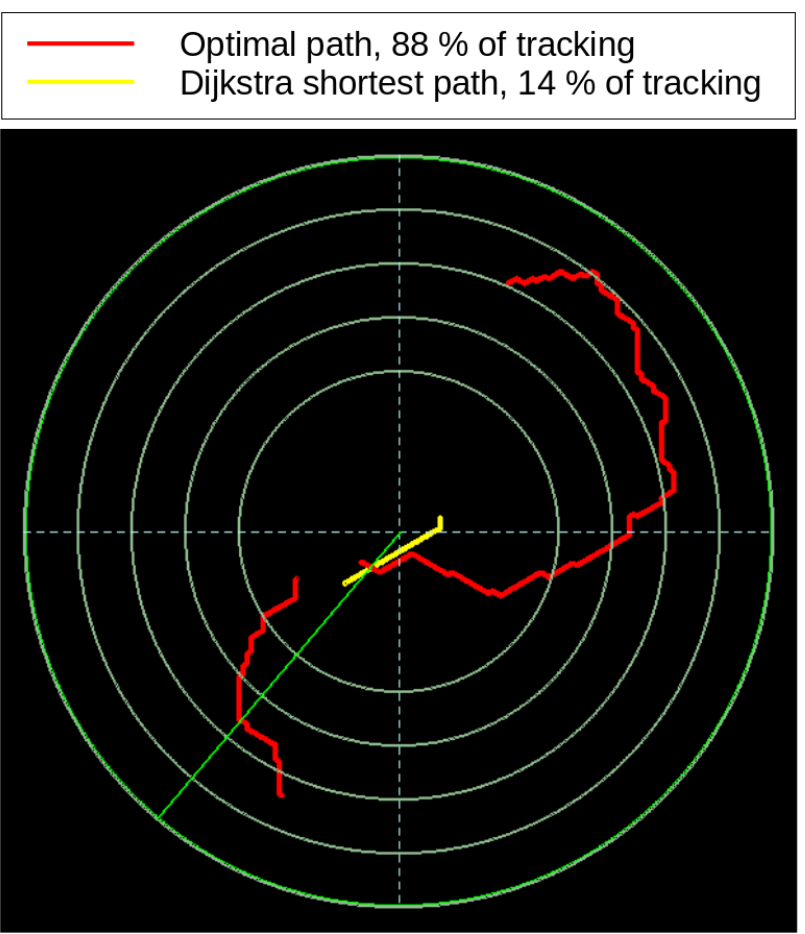

Figure 18. Optimal path Vs Dijkstra shortest path tracking

\section{References}

[1] De Filippis, Luca, and Giorgio Guglieri. Advanced graph search algorithms for path planning of fligh vehicles. INTECH Open Access Publisher, 2012.

[2] De Filippis, L., Guglieri, G., and Quagliotti, F. (2012). Path planning strategies for UAVS in 3D environmen ts. Journal of Intellig ent and Robotic Systems, 65(1-4), 247-264.

[3] Bekhti, Mustapha, Abdennebi, Marw en, Achir, Nadjib, et al. Path planning of unmanned aerial vehicles with terrestrial wireless netw ork tracking. In : 2016 Wireless Days (WD). IEEE, 2016. p. 1-6.

[4] Hart, Peter E., Nils J. Nilsson, and Bertr am Raphael. "A formal basis for the heuristic determina tion of minim um cost paths." Systems Science and Cybernetics, IEEE Transactions on 4.2 (1968): 100-107.

[5] Nilsson, Nils J. Principles of artificia intellig ence. Morgan Kaufmann, 2014.

[6] Podsedkowski, Leszek, et al. "A new solution for path planning in partiall y known or unknown environmen $t$ for nonhol onomic mobile robots." Robotics and Autonomous Systems 34.2 (2001): 145-152.

[7] Kavraki, Lydia E., et al. "Probabilistic roadmaps for path planning in high-dimensional configu ation spaces." Robotics and Automation, IEEE Transactions on 12.4 (1996): 566-580.

[8] LaValle, Steven M., and James J. Kuffner. "Randomized kinodynamic planning. " The International Journal of Robotics Research 20.5 (2001): 378-400.

[9] Wang, Hongl un, et al. "Three-dimensional path planning for unmanned aerial vehicle based on interfered $\mathrm{f}$ uid dynamic system. " Chinese Journal of Aerona utics 28.1 (2015): 229-239. 
[10] Mathew, Neil, Stephen L. Smith, and Steven L. Waslander. "Planning paths for packag e delivery in heterog eneous multirobot teams." IEEE Transactions on Automa tion Science and Engineering 12.4 (2015): 12981308.

[11] Manyam, Satyanar ayana, David Casbeer, and Kaarthik Sundar. "Path Planning for Cooperative Routing of Air-Ground Vehicles." arXiv preprin t arXiv:1605.09739 (2016).

[12] Chen, Yang, Jianda Han, and Xing ang Zhao. "Threedimensional path planning for unmanned aerial vehicle based on linear programming. " Robotica 30.05 (2012): 773-781.

[13] jun2003pa th, title $=\mathrm{P}$ ath planning for unmanned aerial vehicles in uncertain and adversarial environmen ts, author=J un, Myungsoo and DAndrea, Raffaell o, booktitle $=\mathrm{C}$ ooper ative control: models, applica tions and algorithms, pag es=95-110, year=2003, publisher=Spring er
[14] Peng, Xing guang, and Demin Xu. "Intellig ent online path planning for UAVs in adversarial environmen ts." Int J Adv Rob Syst 9 (2012): 1-12.

[15] Ferguson, Dave, Maxim Likhachev, and Anthony Stentz. "A guide to heuristic-based path planning. " Proceedings of the international workshop on planning under uncertain ty for autonomous systems, interna tional conference on automa ted planning and sched uling (ICAPS). 2005.

[16] Yanmaz, Ev?en, et al. "On path planning strategies for netw orked unmanned aerial vehicles." Computer Comm unica tions Workshops (INFOCOM WKSHPS), 2011 IEEE Conference on. IEEE, 2011.

[17] Roberts, James F., Jean-Christophe Zufferey, and Dario Floreano. "Energy manag ement for indoor hovering robots." 2008 IEEE/RSJ Interna tional Conference on Intellig ent Robots and Systems. IEEE, 2008. 